\title{
QUANTITATIVE NULLHOMOTOPY AND RATIONAL HOMOTOPY TYPE
}

\author{
GREGORY R. CHAMBERS, FEDOR MANIN, AND SHMUEL WEINBERGER
}

\begin{abstract}
In Gro14, Gromov asks the following question: given a nullhomotopic map $f: S^{m} \rightarrow$ $S^{n}$ of Lipschitz constant $L$, how does the Lipschitz constant of an optimal nullhomotopy of $f$ depend on $L, m$, and $n$ ? We establish that for fixed $m$ and $n$, the answer is at worst quadratic in $L$. More precisely, we construct a nullhomotopy whose thickness (Lipschitz constant in the space variable) is $C(m, n)(L+1)$ and whose width (Lipschitz constant in the time variable) is $C(m, n)(L+1)^{2}$.

More generally, we prove a similar result for maps $f: X \rightarrow Y$ for any compact Riemannian manifold $X$ and $Y$ a compact simply connected Riemannian manifold in a class which includes complex projective spaces, Grassmannians, and all other simply connected homogeneous spaces. Moreover, for all simply connected $Y$, asymptotic restrictions on the size of nullhomotopies are shown to be determined by rational homotopy type.
\end{abstract}

\section{Contents}

1. Introduction

2. Preliminaries

3. The case of maps $S^{3} \rightarrow S^{2}$

4. Lifting through $k$-invariants

5. Rational invariance

1

6. Proof of the main theorem

7. Some lower bounds

References

\section{INTRODUCTION}

Rational homotopy theory, as introduced by Quillen and Sullivan, is one of the great successes of twentieth-century algebraic topology. It allows one to turn any simply connected space, which may be given as a Postnikov tower or a cell complex, in a rather simple algorithmic way, into one of several, ultimately equivalent, algebraic structures. Moreover, as long as one is willing to ignore torsion, this conversion preserves all homotopic information: it is an equivalence of (rational homotopy) categories.

As in other such cases, we often understand very little about the geometry of the maps that rational homotopy theory tells us must exist. Nevertheless, quite a bit of geometric information may be squeezed out of this algebraic story.

Perhaps the earliest theorem of quantitative algebraic topology is the following, stated by Gromov in Gro78]:

Theorem 1.1. Let $X$ and $Y$ be compact simply connected Riemannian manifolds. Then

$$
\#\{[f] \in[X: Y]: \operatorname{Lip} f \leq L\}=O\left(L^{\alpha}\right),
$$

where $\alpha$ depends only on the rational homotopy type of $X$ and $Y$. 
Naïvely, rational homotopy type should play a role here because torsion homotopy groups can only affect the number of maps by a finite multiplicative constant. But in fact, the proof of this theorem relies heavily on the Sullivan model of rational homotopy theory and its realization via differential forms.

Later on, Gromov Gro99 conjectured that an analogous result should hold for homotopies between maps. To state the conjecture, we first introduce some terminology. Suppose that $X$ and $Y$ are two metric simplicial complexes, and that $f, g: X \rightarrow Y$ are two homotopic maps. We say a homotopy $H: X \times[0,1] \rightarrow Y$ from $f$ to $g$ has thickness $A$ and width $B$ if $d(H(x, t), H(y, t)) \leq$ $A d(x, y)$ for all $x, y$, and $t$ and $d(H(x, t), H(x, s)) \leq B|t-s|$ for all $x, t$, and $s$.

Gromov's original conjecture concerned only the thickness of homotopies:

Conjecture 1. Let $X$ and $Y$ be compact Riemannian manifolds (or some other "reasonable" class of compact metric spaces) with $Y$ simply connected. If $f, g: X \rightarrow Y$ are homotopic maps with Lipschitz constant $\leq L$, then there is a homotopy between them of thickness $O\left(L^{p}\right)$, for some $p$ depending only on the rational homotopy type of $Y$. Perhaps $p$ can always be taken to be 1 .

In FW], Ferry and Weinberger suggest a related problem: can the Lipschitz constant of a homotopy, seen as a map $X \times[0,1] \rightarrow Y$, be bounded linearly in terms of Lip $f$ and $\operatorname{Lip} g$ ? As we show in [CDMW], this is not the case in general. However, one may hope for a polynomial result. In light of the result in this paper, it may be worthwhile to consider thickness and width separately. A compelling if somewhat optimistic conjecture is as follows:

Conjecture 2. In the setting of Conjecture 1, if $f, g: X \rightarrow Y$ are homotopic maps with Lipschitz constant $\leq L$, then there is a homotopy between them of thickness $O(L)$ and width $O\left(L^{p}\right)$, where $p$ depends only on the rational homotopy type of $Y$.

In the case of nullhomotopic maps, we make a stronger conjecture which refers explicitly to the rational homotopy type of $Y$ :

Conjecture 3. If $f: X \rightarrow Y$ is nullhomotopic with Lipschitz constant $\leq L$, then it has a nullhomotopy of thickness $O(L)$ and width $O\left(L^{q}\right)$, where $q$ is the minimal depth of a filtration $0=V_{0} \subset V_{1} \subset \cdots \subset V_{q}$ of the indecomposables in dimensions $\leq n$ of the Sullivan minimal model of $Y$ with the property that $d V_{i} \subseteq \mathbb{Q}\left\langle V_{i-1}\right\rangle$.

The cases $q=0$ and $q=1$ of this conjecture are proved in [FW] and [CDMW], respectively. Indeed, those results hold for homotopies and not only nullhomotopies. In this paper we prove the case $q=2$ :

Theorem 1.2. Let $X$ and $Y$ be finite simplicial complexes, with $X$ n-dimensional. If $Y$ is simply connected and the indecomposables in dimensions $\leq n$ of its Sullivan minimal model split as $V_{1} \oplus V_{2}$ with $d V_{1}=0$ and $d V_{2} \subset \mathbb{Q}\left\langle V_{1}\right\rangle$, then there is a constant $C(X, Y)$ such that nullhomotopic $L$ Lipschitz maps from $X$ to $Y$ admit nullhomotopies of thickness $C(L+1)$ and width $C(L+1)^{2}$.

The class of target spaces covered by this theorem includes, most notably, all simply connected homogeneous spaces, including spheres. As a corollary, when the domain is a suspension, this allows us to find short homotopies, not just nullhomotopies:

Corollary 1.3. In the setting of Theorem [1.2, if in addition $X$ has the homotopy type of a suspension, there is a constant $C^{\prime}(X, Y)$ such that any two homotopic L-Lipschitz maps $f, g: X \rightarrow Y$ have a homotopy of thickness $C^{\prime}(L+1)$ and width $C^{\prime}(L+1)^{2}$.

In particular, this gives a result for maps between spheres. In [Gro14, §2], Gromov asks the following related question: given an $L$-Lipschitz nullhomotopic map $f: S^{m} \rightarrow S^{n}$, how can the Lipschitz constant of a nullhomotopy be bounded as a function of $m, n$ and $L$ ? In this paper we 
get a bound of the form $C(m, n) L^{2}$. Getting an explicit estimate for $C(m, n)$ is a topic for future work which is likely to require some geometric understanding of homotopy groups of spheres.

As further confirmation that rational homotopy type plays a role, we prove the following theorem, which holds for all simply connected targets:

Theorem 1.4. Suppose $X, Y$, and $Z$ are finite complexes, with $X n$-dimensional, and suppose that $Y$ and $Z$ are simply connected and rationally homotopy equivalent. Then nullhomotopic maps $X \rightarrow Y$ and $X \rightarrow Z$ admit nullhomotopies of the same shapes.

We make this more precise below, but for example, if one has certain bounds on thickness and width for nullhomotopies of maps to $Y$, then the same asymptotic bounds hold for maps to $Z$. It would be surprising if this didn't hold for all homotopies rather than just nullhomotopies, but our proof does not generalize.

This paper is in large part a sequel to [CDMW]. While we explicitly restate all the definitions and results we are using from that paper, the reader who has absorbed its main techniques will have an easier time with the more complicated cases covered here.

1.1. The role of rational homotopy. The main geometric tool that we use to construct quantitative nullhomotopies can be seen in a simple example covered by the results in [CDMW]: maps $S^{n} \rightarrow S^{n}$. To nullhomotope such a map, it is enough to cancel point preimages with opposite local degree; this is an idea that goes back to Brouwer. Tracing these point preimages through the nullhomotopy gives an embedded 1-manifold in $S^{n} \times[0,1]$. In order to make the nullhomotopy quantitative, we break up $S^{n} \times[0,1]$ into a grid and make sure that each cube in the grid doesn't "see" too much of this 1-manifold.

Poincaré duality turns this story about 0 - and 1-submanifolds into one about bounded $n$ - and $(n-1)$-dimensional obstruction cochains which generalizes to a result for maps $X \rightarrow K(\mathbb{Z}, n)$ for any finite simplicial complex $X$. In order to generalize this to a larger class of target spaces, one may try to iterate this process over the stages of a Postnikov system, using the obstruction theory for principal fibrations.

As pointed out by Gromov, tracing constants through the way such a lift is built traditionally gives a Lipschitz constant which is a tower of exponentials. Thus to get a reasonable quantitative estimate, we need to once again do the lifting in a local way. Unfortunately, there is no guarantee that the nullhomotopy we came up with in the previous stage is anywhere close to something that lifts. For example, suppose that we are trying to nullhomotope an $L$-Lipschitz map $f: S^{3} \rightarrow S^{2}$ and we have come up with a nullhomotopy $F: S^{3} \times[0,1] \rightarrow \mathbb{C P}^{2}$ which is cellular on a subdivision of a cell structure $S^{3} \times[0,1]$ at scale $1 / L$. We would like to retract this $F$ to a nullhomotopy $S^{3} \times[0,1] \rightarrow S^{2}$. But a priori, the map $\left.F\right|_{t=1 / 2}: S^{3} \rightarrow S^{2}$ need not be nullhomotopic inside of $S^{2}$; by assumption, it is only nullhomotopic as a map to $\mathbb{C} \mathbf{P}^{2}$. Indeed, unless our construction of $F$ was particularly clever, it may have Hopf invariant on the order of $L^{4}$. This means it cannot be made nullhomotopic even after a homotopy in $\mathbb{C P}^{2}$ if that homotopy is to be kept uniformly bounded.

Our (partial) solution to this problem is to turn to algebra. Let $Y$ be a compact metric simplicial complex. Its algebra of $\mathrm{PL}$ forms $A^{*}(Y)$ has a Sullivan minimal model: a differential graded algebra (DGA) $\mathcal{M}^{*}(Y)$ which efficiently encodes the rational homotopy theory of the space and which is realized by a map $R_{Y}: \mathcal{M}^{*}(Y) \rightarrow A^{*}(Y)$ inducing an isomorphism on cohomology. In particular, given a nullhomotopic map $f: X \rightarrow Y$, the map $f^{*} \circ R_{Y}: \mathcal{M}^{*}(Y) \rightarrow A^{*}(X)$ is algebraically nullhomotopic, that is, there is a homomorphism of DGAs

$$
h: \mathcal{M}^{*}(Y) \rightarrow A^{*}(X) \otimes \mathbb{Q}\langle t, d t\rangle
$$

with $\left.h\right|_{t=0, d t=0}=f^{*} \circ R_{Y}$ and $\left.h\right|_{t=1, d t=0}=0$. In particular, this homomorphism must commute with the differential, given on the codomain by a graded Leibniz rule. 
To construct such a homomorphism, one needs to antidifferentiate certain forms. For example, if $X=S^{3}$ and $Y=S^{2}$, then $\mathcal{M}^{*}(Y)$ is given by $\left\langle x_{2}, y_{3} \mid d x=0, d y=x^{2}\right\rangle$ and $R_{Y}$ takes $x$ to a volume form $\omega$ on $S^{2}$ and $y$ to 0 . Then given a nullhomotopic map $f: S^{3} \rightarrow S^{2}$ which is simplicial on a triangulation of $S^{3}$ at scale $1 / L$, a nullhomotopy $\tilde{h}: \mathcal{M}^{*}\left(S^{2}\right) \rightarrow A^{*}\left(S^{3}\right) \otimes \mathbb{Q}\langle t, d t\rangle$ can be given by

$$
\begin{aligned}
& x \mapsto f^{*} \omega \otimes(1-t)^{2}-\alpha \otimes 2(1-t) d t \\
& y \mapsto \eta \otimes 4(1-t)^{3} d t,
\end{aligned}
$$

where $\alpha$ and $\eta$ are defined so as to satisfy $d \alpha=f^{*} \omega$ and $d \eta=f^{*} \omega \wedge \alpha$. Here, $f^{*} \omega \wedge \alpha$ is an exact form since the Hopf invariant of $f$ is zero, using J.H.C. Whitehead's definition of the Hopf invariant via integrals. A filling inequality allows us to choose an $\alpha$ of $\infty$-norm $O(L)$ and-since $\alpha$ and $f^{*} \omega$ each contribute a factor of $L$-an $\eta$ of $\infty$-norm $O\left(L^{2}\right)$.

(Note that the various polynomials in $t$ can be replaced by other polynomials, or even, once we leave purely algebraic territory, by any functions of $t$ which satisfy the differential equations induced by the requirements on $\tilde{h}$. The choice of these functions affects our final estimates only up to a constant.)

By "evaluating" $t$ and $d t$ we can turn this nullhomotopy into a map $h: \mathcal{M}^{*}\left(S^{2}\right) \rightarrow A^{*}\left(S^{3} \times I\right)$; this notation tacitly assumes a simplicial structure on $S^{3} \times I$ whose choice may depend on $L$. If we choose a fine enough subdivision of the interval, into $O\left(L^{2}\right)$ pieces, so that simplices are very skinny in the time direction, then $d t$ is small enough that the integrals over simplices of $h(x)$ and $h(y)$ are bounded uniformly, independent of $L$.

Consider now the previously constructed nullhomotopy $F: S^{3} \times[0,1] \rightarrow \mathbb{C P}^{2}$, and let $\xi$ be a differential form representing the fundamental class of $\mathbb{C} \mathbf{P}^{2}$. If $F^{*} \xi$ is a bounded distance from $h(x)$, then Hopf invariants on boundaries of 4-cells can be determined by integrating a form a bounded distance from $h(y)$. Combined with ideas from [FW] and [CDMW], this allows us to kill these Hopf invariants by modifying the map in a bounded way.

Unfortunately, if we try to continue this process to a third level and beyond, the "errors" are no longer uniformly bounded. This is related to the well-known fact that $(L+1)^{2} \neq L^{2}+1$. This is why what seems to be the second step of an induction does not actually generalize to a proof Conjecture 3 for any $q \geq 3$. At this time we have to be content with Theorem 1.2 .

Another issue with potentially extending this method is that the property of $\tilde{h}$ that one can cancel out large antiderivatives by making $d t$ small is special: one can only construct such a nullhomotopy when $Y$ has positive weights, that is, essentially when $\mathcal{M}^{*}(Y)$ has lots of automorphisms. This property is discussed in [BMSS] and examples of spaces which do not have it are given in [MiT] and Amann. For more general spaces, such nullhomotopies may necessarily have large terms which are not multiples of $d t$. Thus, if one is to find a counterexample to Conjecture 3 in which a nullhomotopy must necessarily have nonlinear thickness, spaces which do not admit positive weights seem to be a natural place to look.

1.2. Optimality. One may ask to what extent our results are sharp. We produce two main examples to this effect. First, we give a sequence of examples, also mentioned in [CDMW], which demonstrate that a linear bound does not always hold, and in some cases the quadratic bound on width is the best we can do. More generally, this family of examples demonstrates that, at least in some cases, the conjectured upper bound of Conjecture 3 is also a lower bound. Secondly, we construct an example which shows that the statement of Theorem 1.2 does not hold if we replace nullhomotopies by homotopies: the exponents in Conjectures 2 and 3 are necessarily different.

Nevertheless, many open questions remain even in the restricted domain of Theorem 1.2. There is some indication that for maps $S^{3} \rightarrow S^{2}$ our quadratic bound on widths of nullhomotopies is not 
sharp, but rather is an artifact of the algebraic method: it is possible to construct nullhomotopies with subquadratic, perhaps even linear Lipschitz constant.

1.3. Outline of the paper. In section 2, we introduce and summarize some geometric results and terminology. This is followed in the third section by a proof of Theorem [1.2 in the special case of maps $S^{3} \rightarrow S^{2}$. Section 4 repeats this for a more general, but still restricted situation. In section 5 we prove that the asymptotic geometry of nullhomotopies is rationally invariant, and section 6 uses this as well as the result of section 4 to prove the main theorem. Finally, in section 7 we discuss lower bounds on the size of homotopies.

1.4. Acknowledgments. The authors would like to thank Dominic Dotterrer for many useful conversations and suggestions over the course of the development of this project, and Mike Freedman for insightful questions that we believe helped improve the exposition. We are grateful to the anonymous referee for many corrections and suggestions for clarifying the exposition. The second author would like to thank Alex Nabutovsky for pointing out certain prior work and related problems.

The first author was partially supported by an NSERC postdoctoral fellowship.

\section{Preliminaries}

In this section, we summarize the geometric machinery developed in [CDMW] as well as introducing some of our own.

2.1. Simplicial approximation and mosaic maps. One result we will make heavy use of is a quantitative simplicial approximation theorem, allowing us to approximate any map between simplicial complexes by a simplicial one with a similar Lipschitz constant. First, we need to define the appropriate kind of subdivision.

Definition. Define a simplicial subdivision scheme to be a family, for every pair of natural numbers $n$ and $L$, of metric simplicial complexes $\Delta^{n}(L)$ isometric to the standard $\Delta^{n}$ with length 1 edges, such that $\Delta^{n}(L)$ restricts to $\Delta^{n-1}(L)$ on all faces. A subdivision scheme is regular if for each $n$ there is a constant $A_{n}$ such that $\Delta^{n}(L)$ has at most $A_{n}$ isometry classes of simplices and a constant $r_{n}$ such that all 1-simplices of $\Delta^{n}(L)$ have length in $\left[r_{n}^{-1} L^{-1}, r_{n} L^{-1}\right]$.

Given a regular subdivision scheme, we can define the $L$-regular subdivision of any metric simplicial complex, where each simplex is replaced by an appropriately scaled copy of $\Delta^{n}(L)$.

Note that $L$ times barycentric subdivision is not regular. Two known examples of regular subdivision schemes are the edgewise subdivision described in EdGr and the cubical subdivision described in [FW].

Proposition 2.1 (Quantitative simplicial approximation theorem). For finite simplicial complexes $X$ and $Y$ with piecewise linear metrics, there are constants $C$ and $C^{\prime}$ such that any L-Lipschitz map $f: X \rightarrow Y$ has a $C L$-Lipschitz simplicial approximation via a homotopy of thickness $C L+C^{\prime}$ and width $C^{\prime}$.

As in [CDMW], we will use simplicial approximation mainly as a way of ensuring that our maps have a uniformly finite number of possible restrictions to simplices. The property that we really care about, then, is the following:

Definition. Let $\mathcal{F}_{k}$ be a finite set of maps $\Delta^{k} \rightarrow Y$, for some space $Y$. If $X$ is a simplicial complex, a map $f: X \rightarrow Y$ is $\mathcal{F}$-mosaic if all of its restrictions to $k$-simplices are in $\mathcal{F}_{k}$. More generally, we can take $X$ to be any polyhedral complex with a finite collection of cell shapes (e.g. a cubical complex, or a product of simplicial complexes) and $\mathcal{F}_{k}$ to be a set of maps from each of the various shapes. 
We refer to a collection of maps as uniformly mosaic if they are all $\mathcal{F}$-mosaic with respect to a fixed unspecified $\mathcal{F}$.

The $\mathcal{F}_{k}$ in the definition naturally form a semi-simplicial set $\mathcal{F}$ via restriction maps. Thus we can think of an $\mathcal{F}$-mosaic map $f$ equivalently as one that factors through

$$
X \stackrel{g}{\rightarrow} \mathcal{F} \stackrel{h_{\mathcal{F}}}{\longrightarrow} Y
$$

where $h_{\mathcal{F}}$ is fixed and $g$ is simplicial, or more generally takes cells isomorphically to cells. In particular, the property of a collection of maps being uniformly mosaic is preserved by postcomposition with any map, for example one collapsing certain simplices.

2.2. Isoperimetry for cochains. In rational homotopy theory, algebraic nullhomotopies are constructed by antidifferentiating certain exact differential forms. To imitate this construction geometrically, we need to be able to antidifferentiate simplicial cochains in a quantitative way. This is given to us by the following lemma, proven in [CDMW]. Here, the $\ell^{\infty}$ norm of a cochain is simply the maximum of its values on simplices.

Lemma 2.2 ( $\ell^{\infty}$ coisoperimetry). Let $X$ be a finite simplicial complex equipped with the standard metric, and let $X_{L}$ be the cubical or edgewise L-regular subdivision of $X$, and $k \geq 1$. Then there is a constant $C_{\mathrm{IP}}=C_{\mathrm{IP}}(X, k)$ such that for any simplicial coboundary $w \in C^{k}\left(X_{L} ; \mathbb{Z}\right)$, there is an $a \in C^{k-1}\left(X_{L} ; \mathbb{Z}\right)$ with $\delta a=w$ such that $\|a\|_{\infty} \leq C_{\mathrm{IP}} L\|w\|_{\infty}$.

The proof of this fact uses the following lemma which we will also need independently.

Lemma 2.3. With the same assumptions, there is a constant $K(X, k)$ such that for any real simplicial cocycle $w \in C^{k-1}\left(X_{L}, \mathbb{R}\right)$, there is an integral cocycle $\tilde{w} \in C^{k-1}\left(X_{L} ; \mathbb{Z}\right)$ with $\|w-\tilde{w}\|_{\infty} \leq$ $K$.

2.3. Quantitative De Rham theory. In order to prove the main theorem, we need to discuss cup products on the cochain level. Since simplicial cup products do not have particularly nice properties, it will be more convenient to use differential forms. Therefore it will be helpful to be able to associate to each simplicial cochain a corresponding standard differential form. We use the notation $\int \omega$ to denote the simplicial cochain obtained by integrating a differential form; here we construct a chain homotopy inverse to this operation.

To do this, we use Whitney's proof of the De Rham theorem, provided in Whi, §IV.27]. Whitney constructs an explicit isomorphism $D$ • from the simplicial cochain complex $C^{\bullet}(M ; \mathbb{R})$ of a manifold $M$ to a subcomplex of $\Omega^{\bullet}(M)$. The same construction produces smooth forms on any simplicial complex as a stratified space. For every $n$, let $\left\{g_{i}^{n}: 0 \leq i \leq n\right\}$ be a smooth partition of unity on the standard simplex $\Delta^{n}=\left\{\vec{x}: \sum_{j=0}^{n} x_{j}=1\right\} \subset \mathbb{R}^{n+1}$ with the following properties:

- $g_{i}^{n} \equiv 1$ near the $i$ th vertex and 0 near the opposite face;

- $\left\{g_{i}^{n}\right\}$ is invariant under the action of the symmetric group;

- for every $j, g_{i}^{n}$ is independent of $x_{j}$ when $x_{j}<\varepsilon_{n}$, for fixed $\varepsilon_{n}>0$;

- $g_{i}^{n} \mid \Delta^{n-1}=g_{i}^{n-1}$.

On any simplicial complex $X$, this defines a smooth partition of unity $\left\{g_{v}: v \in X^{(0)}\right\}$. For a given $\ell$-simplex $c=\left(v_{0}, \ldots, v_{\ell}\right)$, Whitney then defines

$$
D_{\ell}\left(\chi_{c}\right)=\ell ! \sum_{i=0}^{\ell}(-1)^{i} g_{v_{i}} d g_{v_{0}} \wedge \cdots \wedge \widehat{d g_{v_{i}}} \wedge \cdots \wedge d g_{v_{\ell}}
$$

$\left(\phi_{\ell}\right.$ in his notation) and shows that this induces a map $D_{\bullet}: C^{\bullet}(M ; \mathbb{R}) \rightarrow \Omega^{\bullet}(M)$ which is an isomorphism of cochain complexes onto its image.

In order to apply this isomorphism to our situation, we need to make a few more remarks: 
(1) If $p: X \rightarrow Y$ is a simplicial map, then $p^{*} D_{\bullet}=D_{\bullet} p^{*}$. This follows from the special case of an $(n+1)$-simplex collapsed onto an $n$-simplex, which is itself easy to see.

(2) Given two simplicial complexes $X$ and $Y$, the map

$$
D_{\bullet}^{X \times Y}:=\pi_{1}^{*} D_{\bullet}^{X} \wedge \pi_{2}^{*} D_{\bullet}^{Y}: C^{\bullet}(X ; \mathbb{R}) \otimes C^{\bullet}(Y ; \mathbb{R}) \rightarrow \Omega^{\bullet}(X \times Y)
$$

is likewise an isomorphism from the cellular cochains on the product cell structure on $X \times Y$ to its image. In particular, we will use this in the setting $Y=[0,1]$, split into some number of 1-simplices. We will say a form is desimplicial if it is in the image of this map.

(3) Given a Riemannian metric on each stratum of $X$ and a form $\omega$, define $\|\omega\|_{\infty}$ to be the maximum value of $\omega$ on a tuple of unit vectors. Then there are constants $C_{\ell}$ such that if we put on $X \times Y$ the product metric of the standard metrics on simplices, then

$$
\left\|D_{\ell}(c)\right\|_{\infty} \leq C_{\ell}\|c\|_{\infty} \text {. }
$$

(4) Let $\omega \in \Omega^{*}(X \times[0,1])$ be a desimplicial form with $\left.\omega\right|_{X \times\{1\}} \equiv 0$. For a multivector $\xi \in$ $T_{(x, t)}^{n}(X \times I)$, write $\xi_{s}$ for the corresponding multivector in $T_{(x, s)}^{n}(X \times I)$. Then the form

$$
\alpha(\xi)=\int_{t}^{1} \omega_{i}\left(\xi_{s}, d s\right) d s
$$

is also desimplicial.

\section{THE CASE OF MAPS $S^{3} \rightarrow S^{2}$}

In this section, as a warmup, we handle a concrete special case which touches upon most of the problems which we will encounter in proving the more general theorem.

Theorem 3.1. There is a constant $C$ such that any nullhomotopic L-Lipschitz map $f: S^{3} \rightarrow S^{2}$ has a nullhomotopy of width $C(L+1)^{2}$ and thickness $C(L+1)$.

Proof. We first give our spaces some extra structure. We embed $S^{2}$ in $\mathbb{C P}^{2}$, giving each the cell structure with one cell in each even dimension. We give $S^{3}$ a simplicial structure which is an $L$ regular subdivision of some standard one, for example that of $\partial \Delta^{4}$. Finally, let $I=[0,1]$ be given the simplicial structure with $C_{\mathrm{IP}}\left(S^{2}, 2\right) L^{2}$ edges of equal length.

By postcomposing a simplicial approximation with a map contracting simplices, and at the cost of a multiplicative increase in $L$, we can assume that maps $f: S^{3} \rightarrow S^{2}$ are cellular and uniformly mosaic, with restrictions to 2 -simplices having degree between -1 and 1 . We now use a construction similar to that of [CDMW, Thm. 4.2] to construct a complex $\mathcal{G}$ (independent of $f$ ) and a $\mathcal{G}$-mosaic nullhomotopy

$$
F: S^{3} \times I \rightarrow \mathcal{G} \rightarrow \mathbb{C P}^{2} .
$$

Since $f$ is cellular, we can define a cochain $w \in C^{2}\left(S^{3} ; \pi_{2}\left(S^{2}\right)\right)$ by $\langle w, c\rangle=\left[\left.f\right|_{c}\right] \in \pi_{2}\left(S^{2}\right)$. Since $f$ is nullhomotopic, this cochain is the coboundary of some $a \in C^{1}\left(S^{3} ; \pi_{2}\left(S^{2}\right)\right)$. By Lemma 2.2, since $\|w\|_{\infty}=1$, we can pick $a$ such that $\|a\|_{\infty} \leq C_{\mathrm{IP}} L$.

Now let $\hat{a} \in C^{1}\left(S^{3} \times I ; \pi_{2}\left(S^{2}\right)\right)$ be defined by

$$
\begin{aligned}
& \left\langle\hat{a}, v \times\left[\frac{i}{C_{\mathrm{IP}} L^{2}}, \frac{i+1}{C_{\mathrm{IP}} L^{2}}\right]\right\rangle=0 \\
& \text { for 0-simplices } v \text { of } S^{3} \text {, } \\
& 0 \leq i \leq C_{\mathrm{IP}} L^{2} \\
& \left\langle\hat{a}, e \times\left\{\frac{i}{C_{\mathrm{IP}} L^{2}}\right\}\right\rangle=\left\lfloor\left(1-\frac{i}{C_{\mathrm{IP}} L^{2}}\right)^{2}\langle a, e\rangle\right\rfloor \quad \begin{array}{r}
\text { for } 1 \text {-simplices } e \text { of } S^{3} \\
0 \leq i \leq C_{\mathrm{IP}} L^{2}
\end{array}
\end{aligned}
$$

In other words, $\hat{a}$ is the "rounded off" version of the cochain $\bar{a}$ whose value on $e \times\{t\}$ is $\left(1-t^{2}\right)\langle a, e\rangle$. This ensures that the cochain $\delta \hat{a}$ has the following properties:

(1) $\|\delta \hat{a}\|_{\infty} \leq 3 ;$ 
(2) $\left.\delta \hat{a}\right|_{S^{3} \times\{0\}}=w$;

(3) and $\left.\delta \hat{a}\right|_{S^{3} \times\{1\}}=0$.

This allows us to build $F: S^{3} \times I \rightarrow \mathbb{C P}^{2}$ by skeleta as follows. Send the 1-skeleton to the basepoint; this gives us $\mathcal{G}_{0}$ and $\mathcal{G}_{1}$ with one map each. Then send each 2-cell $c$ to $S^{2} \subset \mathbb{C P}^{2}$ via a fixed map of degree $\langle\delta \hat{a}, c\rangle$. This gives us a $\mathcal{G}_{2}$ with one cell per degree between -3 and 3 and shape of cell, and a partial map $F:\left(S^{3} \times I\right)^{(2)}$ which can be extended to the 3 -skeleton with no obstruction since $\delta \hat{a}$ evaluates to zero on cycles. For each possible map on the boundary of a 3-cell, we fix a filling, giving $\mathcal{G}_{3}$ and an extension of $F$ to the 3-skeleton. Since there is no obstruction to extending the map to the 4-skeleton, we again fix a filling for each possible map on the boundary of a 4-cell. At each step, we also include the zero map and the restrictions of $f$ to simplices in the corresponding skeleton of $\mathcal{G}$, and ensure that the restriction to $S^{3} \times\{0,1\}$ is correct. This completes the construction of $F$.

We will proceed by changing this nullhomotopy into one which maps to $S^{2}$. For this to work, we need to kill the Hopf obstruction; that is, to change its behavior on the 3-cells of $S^{3} \times I$ so that the restriction to the the boundary of each 4-cell of $S^{3} \times I$ has Hopf invariant 0 .

Let us translate this into the language of differential forms. The cohomology $\operatorname{ring} H^{*}\left(\mathbb{C P}^{2} ; \mathbb{Z}\right)=$ $\mathbb{Z}[x] /\left(x^{3}\right)$, where $x \in H^{2}\left(\mathbb{C P}^{2} ; \mathbb{Z}\right)$. Let $\xi$ be a differential form representing $x$, with the extra property that $f^{*} \xi$ is desimplicial; this is possible from the restrictions we put on $f$. Then for a 4-cell $p$ of $S^{3} \times I, \int_{p} F^{*} \xi^{2}$ is the degree of $\left.F\right|_{p}$ over the 4-cell of $\mathbb{C P}^{2}$, or equivalently the Hopf invariant of $\left.F\right|_{\partial p}$ (this restriction is a map to $S^{2}$ since $F$ is cellular.) If $\alpha$ is any 1-form with $d \alpha=F^{*} \xi$, then this Hopf invariant is given by Stokes' theorem by $\int_{\partial p} \alpha \wedge F^{*} \xi$. Now suppose we have a cochain $b \in C^{3}\left(S^{3} \times I ; \mathbb{Z}\right)$ such that $\langle b, \partial p\rangle$, or in other words $\delta b=\int F^{*} \xi^{2}$, but which (probably unlike $\int \alpha \wedge F^{*} \xi$ ) takes uniformly bounded, integer values on simplices. This would allow us to construct the new nullhomotopy as follows. Given two maps $u, v$ from a disk (of any dimension $m$ ) to some other space which coincide on the boundary, let $u * v$ denote the map on the $m$-sphere which restricts to $u$ and $v$ on the two hemispheres. Then:

- For each 3-cell $q \in S^{3} \times I$, replace $\left.F\right|_{q}$ with a map $\left.G\right|_{q}$ such that the map $\left.\left.F\right|_{q} * G\right|_{q}: S^{3} \rightarrow S^{2}$ (which behaves like $F$ on the upper hemisphere and $G$ on the lower) has Hopf invariant $\langle b, q\rangle$.

- Extend $G$ to 4-cells; this can be done since the Hopf invariant on the boundary of each 4-cell is zero.

Finding a $b$ which satisfies these properties will be the goal of the rest of the proof.

We note that the behaviors of $F^{*} \xi$ on $k$-cells are in one-to-one correspondence with the set $\mathcal{G}_{k}$. For now, though, instead of $F^{*} \xi$ we will use the desimplicial form $\hat{\omega}:=D_{2} \int F^{*} \xi$. This allows us to define a nice antidifferential.

We write $\omega \in \Omega^{2}\left(S^{3}\right)$ to mean the restriction of $\hat{\omega}$ to $S^{3} \times\{0\}$ (which is also $f^{*} \xi$.) Further on, we will also define a "smooth" interpolation $\bar{\omega}$ between $\omega$ and 0 , as opposed to the "bumpy" interpolation $\hat{\omega}$. Note also that $\omega$ is the "differential form version" of the cochain $w$. We use a similar convention for other forms further on.

For a vector $v \in T_{(x, t)}\left(S^{3} \times I\right)$, write $v_{s}$ for its translate in $T_{(x, s)}\left(S^{3} \times I\right)$. Now, since $\left.\hat{\omega}\right|_{S^{3} \times\{1\}} \equiv 0$, and by the Poincaré lemma, the 1 -form

$$
\hat{\alpha}(v)=\int_{t}^{1} \hat{\omega}\left(d s, v_{s}\right) d s \in \Omega^{1}\left(S^{3} \times I\right)
$$

satisfies $d \hat{\alpha}=\hat{\omega}$ and $\int \hat{\alpha}=\hat{a}$. Moreover, since $\hat{\omega}$ is desimplicial, this also means that $\|\alpha\|_{\infty} \leq L\|\omega\|_{\infty}$ and, as discussed in $₫ 2.3, \alpha$ is desimplicial. Thus we know that $\|\hat{\alpha} \wedge \hat{\omega}\|_{\infty} \leq C L$, but we don't have a constant bound. On the way to defining the desired cochain $b$, we will find a uniformly bounded 
form $\beta$ such that for any 4-cell $p$,

$$
\int_{\partial p} \beta=\int_{\partial p} \hat{\alpha} \wedge \hat{\omega}=\int_{p} \hat{\omega}^{2} .
$$

Even then, we will not be able to simply set $b=\int \beta$, both because $\int \beta$ may not be integral and because $\delta \int \beta=\int \hat{\omega}^{2}$, which is potentially a different cochain from $\int F^{*} \xi^{2}$. Nevertheless, after $\beta$ is constructed, there is only a short way to go to building $b$.

To construct $\beta$, we recall the algebraic nullhomotopy $\bar{h}: \mathcal{M}^{*}\left(S^{2}\right) \rightarrow A^{*}\left(S^{3}\right) \otimes \mathbb{Q}\langle t, d t\rangle$ from the introduction, given by

$$
\begin{aligned}
& x \mapsto \omega \otimes(1-t)^{2}-\alpha \otimes 2(1-t) d t \\
& y \mapsto \eta \otimes 4(1-t)^{3} d t,
\end{aligned}
$$

where we choose $\alpha$ and $\eta$ so that $\alpha=\left.\hat{\alpha}\right|_{S^{3} \times\{0\}}$ and $d \eta=\alpha \wedge \omega$. Note that our isoperimetric results mean that we can choose $\eta$ to have $\infty$-norm $O\left(L^{2}\right)$. Moreover, since we have subdivided the interval into $O\left(L^{2}\right)$ pieces, $d t$ thought of as a 1-form on this subdivision has $\infty$-norm $O\left(L^{-2}\right)$. Now, $\hat{\omega}^{2}$ can be thought of as an approximation of

$$
\bar{h}\left(x^{2}\right)=d \bar{h}(y)=\alpha \wedge \omega \otimes 4(1-t)^{3} d t .
$$

Therefore we can use the bounded form $\bar{h}(y)=\eta \otimes 4(1-t)^{3} d t$ as a scaffolding to help us build a form with bounded $\infty$-norm whose derivative is $\hat{\omega}^{2}$.

To this end, writing $\pi: S^{3} \times[0,1] \rightarrow S^{3}$ for the projection onto the first factor, we let

$$
\begin{aligned}
& \Delta \alpha:=\hat{\alpha}-\bar{\alpha}, \text { where } \bar{\alpha}=(1-t)^{2} \pi^{*} \alpha \\
& \Delta \omega:=d \Delta \alpha=\hat{\omega}-\bar{\omega}, \text { where } \bar{\omega}=(1-t)^{2} \pi^{*} \omega-2(1-t) d t \wedge \pi^{*} \alpha .
\end{aligned}
$$

In other words, $\Delta \alpha$ is the (bounded!) difference between $\hat{\alpha}$ and the form that $\hat{\alpha}$ would be if we hadn't had to take integer parts in the construction of its cochain counterpart $\hat{a}$. So by construction, $\Delta \alpha$ and $\Delta \omega$ are both bounded.

Now, by Stokes' theorem, for any 4-cell $p$ of $S^{3} \times I$, the Hopf invariant of $F$ on its boundary is given by

$$
\begin{aligned}
\int_{p} \hat{\omega}^{2} & =\int_{p}\left[(\Delta \omega)^{2}+2 \bar{\omega} \wedge \Delta \omega+\bar{\omega}^{2}\right] \\
& =\int_{p}\left[(2 \hat{\omega}-\Delta \omega) \wedge \Delta \omega-4(1-t)^{3} d t \wedge \pi^{*}(\alpha \wedge \omega)\right] \\
& =\int_{\partial p}\left[(2 \hat{\omega}-\Delta \omega) \wedge \Delta \alpha-4(1-t)^{3} d t \wedge \pi^{*} \eta\right] .
\end{aligned}
$$

Here, the equality between the first and second lines holds because $\alpha \wedge \alpha$ and $\omega \wedge \omega$ are both zero.

Call the integrand in the last line $\beta$. We see that both terms of $\beta$ are uniformly bounded and are zero when restricted to $S^{3} \times\{0,1\}$.

Now consider the uniformly bounded cellular cochain $\int \beta \in C^{3}\left(S^{3} \times I ; \mathbb{R}\right)$. We have $\delta \int \beta=\int \hat{\omega}^{2}$, but it may not be the case that $\int \hat{\omega}^{2}$ is the same cochain as $\int F^{*} \xi^{2}$, which is the degree of $F$ on 4-cells. This can be resolved in the following manner. Recall that $F$ factors through maps

$$
S^{3} \times I \stackrel{G}{\rightarrow} \mathcal{G} \stackrel{H}{\rightarrow} \mathbb{C} \mathbf{P}^{2},
$$

where $\mathcal{G}$ is a fixed finite polyhedral complex independent of $L$. Then $\left(D_{2} \int H^{*} \xi\right)^{2}$ and $H^{*} \xi^{2}$ are well-defined, cohomologous forms on $\mathcal{G}$ and thus there is a cellular cochain $\mathfrak{g} \in C^{3}(\mathcal{G} ; \mathbb{R})$, again independent of $L$, such that

$$
d \mathfrak{g}=\int \underset{9}{\left(D_{2} \int H^{*} \xi\right)^{2}-\int H^{*} \xi^{2}}
$$


Then $b^{\prime}=\int \beta-G^{*} \mathfrak{g}$ is a uniformly bounded cochain on $S^{3} \times I$ with $\delta\left(\int \beta-G^{*} \mathfrak{g}\right)=\int F^{*} \xi^{2}$. This cochain is not integral, but it does restrict to zero on $S^{3} \times\{0,1\}$.

By Lemma 2.3, we can find an integral cochain $b_{0} \in C^{2}\left(S^{3} ; \mathbb{Z}\right)$ such that for every 2 -simplex $q$ of $S^{3}$,

$$
\left|\left\langle b_{0}, q\right\rangle-\left\langle b^{\prime}, q \times[0,1]\right\rangle\right| \leq K\left(S^{3}, 3\right) .
$$

We then set $b$ by taking nearest integers to $b^{\prime}$, similarly to how we constructed $\hat{a}$ from $\bar{a}$. Specifically, we set $\left\langle b, q \times\left[(i-1) / C_{\mathrm{IP}} L^{2}, i / C_{\mathrm{IP}} L^{2}\right]\right\rangle$ so that

$$
\left\langle b-b^{\prime}, q \times\left[0, i / C_{\mathrm{IP}} L^{2}\right]\right\rangle \in[0,1),
$$

for $i \neq C_{\mathrm{IP}} L^{2}$ (these values are at most distance 1 from those of $b^{\prime}$ ) and set the values on the last time increment so that $\langle b, q \times[0,1]\rangle=\left\langle b_{0}, q\right\rangle$ (and hence they are at most $K\left(S^{3}, 3\right)+1$ away from those of $\left.b^{\prime}\right)$. This together with the requirement that $\delta b=\int F^{*} \xi^{2}$ fixes the values on the transverse 3 -simplices of $S^{3} \times[0,1]$; these values are at most distance 4 from those of $\beta$. Therefore we get

$$
\|b\|_{\infty} \leq\left\|b^{\prime}\right\|_{\infty}+\max \left\{K\left(S^{3}, 3\right)+1,4\right\} .
$$

This is a uniform bound and completes the proof.

\section{LiFTING THROUGH $k$-INVARIANTS}

We now extend the argument for $S^{3} \rightarrow S^{2}$ to a setting which is still geometrically constrained, but which contains a larger class of rational homotopy types which, together with the rational invariance results in the next section, can be assembled into the final result.

Theorem 4.1. Let $X$ be a finite $N$-dimensional simplicial complex. For $i=1, \ldots, r$, let $n_{i} \geq 2$ and let $B_{i}$ be a finite $C W$ complex with an $(N+1)$-connected map $B_{i} \rightarrow K\left(\mathbb{Z}, n_{i}\right)$ whose $C W$ structure is that of a simplicial complex with the $\left(n_{i}-1\right)$-skeleton collapsed. Define a $C W$-complex $B=\prod_{i=1}^{r} B_{i}$. For some $2 \leq n \leq N$, let $Y$ be a finite subcomplex, whose inclusion map is $(N+1)$ connected, of the total space of a $K(\mathbb{Z}, n)$-fibration over $B$, with projection map $p: Y \rightarrow B$. Then there is a $C(X, Y)$ such that any nullhomotopic L-Lipschitz map $f: X \rightarrow Y$ has a nullhomotopy of width $C(L+1)^{2}$ and thickness $C(L+1)$.

Note that $K(\mathbb{Z}, n)$-fibrations over $B$ are, up to equivalence, in bijection with elements of $H^{n+1}(B)$ which represent the obstruction to constructing a section, and that any such fibration can be made to have finite skeleta, for example using Milnor's construction [Mil].

The proof follows an outline similar to the special case in the previous section. The main differences are technicalities imposed by the need to lift through a fibration where in the last section we retracted.

Proof. Up to dimension $N, H^{*}(B ; \mathbb{Q})$ is naturally isomorphic to a free graded commutative $\mathbb{Q}$ algebra generated by elements of degree $n_{i}$. Suppose first that the primary (and only) obstruction in $H^{n+1}(B ; \mathbb{Q})$ to trivializing $p$ has an indecomposable summand in this algebra. Equivalently, $\pi_{n}$ of the fiber goes to a finite quotient in $Y$, so up to rational homotopy type up to dimension $N, Y$ is still a product of Eilenberg-MacLane spaces. This case follows directly from the main theorem of [CDMW], and linear nullhomotopies can be found; therefore, in the rest of this proof, we assume that this obstruction class is contained in the ideal generated by products in $H^{*}(B ; \mathbb{Q})$.

We start by showing that $f$ can be assumed to take on a certain structure, in particular being uniformly mosaic on a subdivision of $X$ at scale $L$. We will implicitly work with such a subdivision; when we take the $L^{\infty}$ norm of forms on $X$, we will do so with respect to the metric in which the simplices of the subdivision are of unit size.

Let $\pi_{i}$ be the projection $B \rightarrow B_{i}$. We can simplicially approximate a map homotopic to $\pi_{i} \circ p \circ f$ on the distinguished simplicial model of $B_{i}$, then send it back to $B_{i}$ via the map collapsing the 
$\left(n_{i}-1\right)$-skeleton. This gives us a short homotopy between $\pi_{i} \circ p \circ f$ and a cellular map on an $O(L)$ regular subdivision of $X$ which is $\mathcal{F}^{i}$-mosaic for some $\mathcal{F}^{i}$ depending only on $B_{i}$ and the homotopy equivalence. This gives us a short homotopy $E_{t}$ from $p \circ f$ to a $\mathcal{F}$-mosaic map, where $\mathcal{F}_{k}=\prod_{i=1}^{r} \mathcal{F}_{k}^{i}$ and the boundary maps are also products.

Finally, we would like to lift $E_{t}$ to a short homotopy of $f$. By homotopy lifting, this can be done, but we would like it done quantitatively in order to produce a short homotopy $\tilde{E}_{t}$ from $f$ to an $\tilde{\mathcal{F}}$-mosaic map for some $\tilde{\mathcal{F}}$. Therefore we do this by skeleta. For $k<n$, we can choose a unique lift for every $k$-simplex of $\mathcal{F}$. Now let $c$ be an $n$-simplex of $X$. We would like to show that we can lift $\left.E_{t}\right|_{c}$ so that $\left.\tilde{E}_{1}\right|_{c}$ is one of a uniformly finite number of maps.

Let $\tilde{c}=c \times\{0\} \cup \partial c \times[0,1]$. Since $E$ is uniformly Lipschitz with respect to the standard metric on the subdivision, we can simplicially approximate $\left.E\right|_{\tilde{c}}$ at a uniform scale. In particular, if $u: \tilde{c} \times[0,1]$ is the linear homotopy to the simplicial approximation, the map $\left.u\right|_{\tilde{c} \times\{1\} \cup \partial \tilde{c} \times[0,1]}$ takes on a uniformly finite number of values which we include in $\tilde{\mathcal{F}}_{n}$. We can take this to be the map $\left.\tilde{E}_{1}\right|_{c}$.

Finally, for higher skeleta all lifts are again homotopic, and so when $k>n$ we can take a unique lift for every restriction of $\tilde{E}_{t}$ to the boundary of a $k$-cell. The set of such lifts will be called $\tilde{\mathcal{F}}_{k}$.

Now, at the cost of a linear penalty on $L$, we can assume that $f$ is $\tilde{\mathcal{F}}$-mosaic, and therefore each $f_{i}$ is $\mathcal{F}^{i}$-mosaic. For each $f_{i}$, a construction similar to that of the homotopy $F$ in the previous section builds a nullhomotopy $F_{i}: X \times I \rightarrow B_{i}$ to the following specifications.

- $F_{i}$ is $\mathcal{G}^{i}$-mosaic for some $\mathcal{G}^{i}$, again depending only on $B_{i}$, on a cell structure obtained by splitting the interval $I$ into $C_{\ell}^{2} L^{2}$ equal subintervals, where $C_{\ell}=\max _{n \leq N} C_{\mathrm{IP}}(X, n)$;

- The degree of $F_{i}$ on $n_{i}$-cells of this cell structure is as follows. Let $w_{i} \in C^{n_{i}}(X)$ be the cochain whose values are the degrees of $f_{i}$ on simplices, and let $a_{i} \in C^{n_{i}-1}(X)$ be a cochain with $\delta a_{i}=w_{i}$ and $\left\|a_{i}\right\|_{\infty} \leq C_{\mathrm{IP}} L\left\|w_{i}\right\|_{\infty}$. Such an $a_{i}$ exists since $f_{i}$ is nullhomotopic. Then the degree of $F_{i}$ on a cell $c$ is given by $\left\langle\delta \hat{a}_{i}, c\right\rangle$, where $\hat{a}_{i} \in C^{n_{i}-1}(X \times I)$ is defined by

$$
\left\langle\hat{a}_{i}, c \times\{t\}\right\rangle=\left\lfloor(1-t)^{n_{i}}\left\langle\hat{a}_{i}, c\right\rangle\right\rfloor
$$

on cells of that form and is zero on cells which extend in the time direction. Since the derivative of $(1-t)^{n_{i}}$ and the values of $w_{i}$ are uniformly bounded, so are the values of $\delta \hat{a}_{i}$.

Then $F=\left(F_{1}, \ldots, F_{r}\right)$ is a nullhomotopy $F$ of $f$ in $B$ which is $\mathcal{G}$-mosaic, where once again $\mathcal{G}_{k}=$ $\prod_{i=1}^{r} \mathcal{G}_{k}^{i}$. Our plan is to find a nullhomotopy in $Y$ which projects onto $F$, again modeled on an algebraic nullhomotopy

$$
\bar{h}: \mathcal{M}^{*}(Y) \rightarrow \Omega^{*}(X) \otimes \mathbb{Q}\langle t, d t\rangle .
$$

The minimal model of $B$ is a free algebra $\mathcal{M}(B)$ with trivial differential on the $n_{i}$-dimensional generators $x_{i}$ corresponding to the fundamental class of each $B_{i}$. The projection $p: Y \rightarrow B$ corresponds to an extension

$$
p^{*}: \mathcal{M}^{*}(B) \rightarrow \mathcal{M}^{*}(Y)=\mathcal{M}(B) \otimes \mathbb{Q}\langle y\rangle,
$$

where $d y=P\left(x_{1}, \ldots, x_{r}\right)$ is the aforementioned cohomological obstruction in $\mathbb{Q}\left\langle x_{1}, \ldots, x_{r}\right\rangle$ to finding a section of $Y$; this is a polynomial all of whose terms have total degree at least 2 .

For each $i$, let $\xi_{i} \in \Omega^{n_{i}}\left(B_{i}\right)$ be a form representing the fundamental class of $B_{i}$. We then write

$$
\omega_{i}=D_{n_{i}} \int f^{*} p^{*} p_{i}^{*} \xi_{i} .
$$

Note that since $f_{i}$ is a composition of a simplicial map and a collapse, $\omega_{i}$ is the pullback of a desimplicial form $\xi_{i}^{\prime}$ representing the fundamental class in $H^{n_{i}}\left(K\left(\mathbb{Z}, n_{i}\right)\right)$. Thus we can find a form $\nu=f^{*} \zeta$ where

$$
d \zeta=P\left(p_{1}^{*} \xi_{1}^{\prime}, \ldots, p_{r}^{*} \xi_{r}^{\prime}\right)
$$


(Further on, we will write this as $P\left(\overrightarrow{p_{-}^{*} \xi^{\prime}}\right)$.) Since $\zeta$ doesn't depend on $f$ or $X,\|\nu\|_{\infty}$ is uniformly bounded, as are the $\omega_{i}$. Similar to the previous section, we have that for an $(n+1)$-simplex $p$ of $X$,

$$
\int_{\partial p} \nu=\int_{p} P(\vec{\omega}) \neq \int_{p} P\left(\overrightarrow{f^{*} p^{*} p_{-}^{*} \xi}\right)
$$

but they differ by a small coboundary and we will later need to take this into account.

We therefore get a homomorphism $\bar{f}: \mathcal{M}^{*}(Y) \rightarrow \Omega^{*}(X)$ defined by $x_{i} \mapsto \omega_{i}$ and $y \mapsto \nu$. Since $f$ is nullhomotopic, we can build the algebraic nullhomotopy $\bar{h}$ of $\bar{f}$ as follows. For any DGA $A$ define an operator $\int_{0}^{1}: A \otimes\langle t, d t\rangle \rightarrow A$ by

$$
\int_{0}^{1} a \otimes t^{i}=0, \int_{0}^{1} a \otimes t^{i} d t=(-1)^{\operatorname{deg} a} \frac{a}{i+1} .
$$

Then send

$$
\begin{aligned}
x_{i} & \mapsto \omega_{i} \otimes(1-t)^{n_{i}}+(-1)^{n_{i}+1} \alpha_{i} \otimes n_{i}(1-t)^{n_{i}-1} d t \\
y & \mapsto \nu \otimes(1-t)^{n+1}+\eta \otimes(n+1)(1-t)^{n} d t,
\end{aligned}
$$

where $\alpha$ and $\eta$ are chosen so that $d \alpha_{i}=\omega_{i}$ and $d \eta=\int_{0}^{1} \bar{h}(P(\vec{x}))+(-1)^{n+1} \nu$.

Note that the terms of $\int_{0}^{1} \bar{h}(P(\vec{x}))$ are each a product of some $\omega_{i}$ 's together with one $\alpha_{i}$. Since $\alpha_{i}$ may be chosen so that $\left\|\alpha_{i}\right\|_{\infty}=O(L)$, this means that $\eta$ may be chosen so that $\|\eta\|_{\infty}=O\left(L^{2}\right)$.

On the other hand, define a form $\hat{\omega}_{i}=D_{n_{i}} \int F_{i}^{*} p_{i}^{*} \xi_{i} \in \Omega^{n_{i}}(X \times[0,1])$. This gives us a homomorphism $\bar{F}: \mathcal{M}(B) \rightarrow \Omega^{*}(X \times[0,1])$.

Write $\pi: X \times[0,1] \rightarrow X$ for the projection onto the first factor. For a multivector $\xi \in T_{(x, t)}^{n}(X \times I)$, and writing $\xi_{s}$ for its parallel translate in $T_{(x, s)}^{n}(X \times I)$, let

$$
\hat{\alpha}_{i}(\xi)=\int_{t}^{1} \omega_{i}\left(d s, \xi_{s}\right) d s .
$$

Then $d \hat{\alpha}_{i}=\hat{\omega}_{i}$. Now defining forms $\Delta \alpha_{i}, \Delta \omega_{i}, \bar{\alpha}_{i}$ and $\bar{\omega}_{i}$ by

$$
\begin{aligned}
\Delta \alpha_{i} & :=\hat{\alpha}_{i}-\bar{\alpha}_{i}:=\hat{\alpha}_{i}-(1-t)^{n_{i}} \pi^{*} \alpha_{i} \\
\Delta \omega_{i} & :=d \Delta \alpha_{i}=\hat{\omega}_{i}-\bar{\omega}_{i} \\
& :=\hat{\omega}_{i}-(1-t)^{n_{i}} \pi^{*} \omega_{i}-n_{i}(1-t)^{n_{i}-1} d t \wedge \pi^{*} \alpha_{i},
\end{aligned}
$$

we get $\left\|\Delta \omega_{i}\right\|_{\infty} \leq C(N, B)$ and $\left\|\Delta \alpha_{i}\right\|_{\infty} \leq C(N, B)$.

Now, by Stokes' theorem, for every $(n+1)$-cell $c$ of $X \times[0,1]$,

$$
\begin{aligned}
\int_{c} \bar{F}(P(\vec{x})) & =\int_{c} P(\overrightarrow{\Delta \omega}-\overrightarrow{\vec{\omega}}) \\
& =\int_{c}\left[\sum_{i} \Delta \omega_{i} \operatorname{poly}(\overrightarrow{\Delta \omega}, \overrightarrow{\vec{\omega}})-P(\overrightarrow{\vec{\omega}})\right] \\
& =\int_{\partial c}\left[\sum_{i} \Delta \alpha_{i} \operatorname{poly}(\overrightarrow{\Delta \omega}, \vec{\omega})-(1-t)^{n+1} \pi^{*} \nu-(n+1)(1-t)^{n} \pi^{*} \eta \wedge d t\right] .
\end{aligned}
$$

Call the integrand in the previous line $\beta$. Then since $\|d t\|_{\infty}=1 / C_{\ell}^{2} L^{2}$ and the polynomials we have elided can be chosen so as to depend only on $P, \beta$ satisfies $\|\beta\|_{\infty} \leq C(N, Y)$ and $d \beta=P(\vec{\omega})$.

Now we are ready to construct a lift $\tilde{F}: X \times I \rightarrow Y$ of $F$. Since $F$ is $\mathcal{G}$-mosaic, we can view it as a composition

$$
X \times I \stackrel{G}{\rightarrow} \mathcal{G} \stackrel{H}{\rightarrow} B
$$


Now let $\mathcal{G}^{\prime}$ be the smallest complex which surjects onto $\mathcal{G}$ and such that $\tilde{\mathcal{F}}$ in turn injects into it, with the composition induced by the projection $\tilde{\mathcal{F}} \rightarrow \mathcal{F}$. In particular, $\left(\mathcal{G}^{\prime}\right)^{(n-1)}=\mathcal{G}^{(n-1)}$ but in the $n$-skeleton, some cells have a number of duplicates compared to $\mathcal{G}$. Then there are obvious maps

$$
X \times I \stackrel{G^{\prime}}{\longrightarrow} \mathcal{G}^{\prime} \stackrel{H^{\prime}}{\longrightarrow} B .
$$

We can build a partial lift of $H^{\prime}$ to $Y$ by lifting each map in $\mathcal{G}_{k}^{\prime}$, for each $k \leq n$, using the lift in $\tilde{\mathcal{F}}$ where it exists. This then gives us a map $\tilde{H}:\left(\mathcal{G}^{\prime}\right)^{(n)} \rightarrow Y$ and an obstruction cocycle $\mathfrak{o} \in C^{n+1}\left(\mathcal{G}^{\prime}, \tilde{\mathcal{F}} ; \mathbb{Z}\right)$ to extending it to $\left(\mathcal{G}^{\prime}\right)^{(n+1)}$ which is independent of $f$.

Now, $\mathfrak{o}$ and $P\left(D_{2} \int\left(H^{\prime}\right)^{*} p^{*} p_{i}^{*} \xi_{i}\right)$ are both representatives of the obstruction class in $H^{n+1}\left(\mathcal{G}^{\prime}, \tilde{\mathcal{F}} ; \mathbb{R}\right)$ to lifting $H$ to a map $\mathcal{G} \rightarrow Y$. Therefore, there is a cellular cochain $\mathfrak{a} \in C^{n}\left(\mathcal{G}^{\prime}, \tilde{\mathcal{F}} ; \mathbb{R}\right)$ such that

$$
\delta \mathfrak{a}=\int P\left(D_{2} \int\left(H^{\prime}\right)^{*} p^{*} p_{i}^{*} \xi_{i}\right)-\mathfrak{o} .
$$

Since

$$
\overline{F^{*}} P(\vec{x})=\left(G^{\prime}\right)^{*} P\left(D_{2} \int\left(H^{\prime}\right)^{*} p^{*} p_{i}^{*} \xi_{i}\right),
$$

the uniformly bounded cochain $\int \beta-\left(G^{\prime}\right)^{*} \mathfrak{a} \in C^{n}(X \times I ; \mathbb{R})$ satisfies

$$
\delta\left(\int \beta-\left(G^{\prime}\right)^{*} \mathfrak{a}\right)=\left(G^{\prime}\right)^{*} \mathfrak{o} .
$$

This cochain is not integral, but we can use the method in the previous section to find a nearby integral cochain $\mathfrak{b}$ with the same coboundary, and such that it is still zero on $X \times\{0,1\}$.

We will use $\mathfrak{b}$ to construct a lift of $F$ to $Y$ which is $\tilde{\mathcal{G}}$-mosaic for a $\tilde{\mathcal{G}}=\tilde{\mathcal{G}}(X, Y)$ which we first construct. Let $\tilde{\mathcal{G}}$ contain $\tilde{\mathcal{F}}$ and for $k \leq n-1$ let $\tilde{\mathcal{G}}_{k}$ consist of the $\tilde{H}$-lifts of $\mathcal{G}_{k}^{\prime}$. Next, for every value $\gamma$ that may be taken by $\mathfrak{b}$ and every element $\delta \in \mathcal{G}_{n}^{\prime} \backslash \tilde{\mathcal{F}}_{n}$ we add in a lift $\ell(\delta, \gamma)$ which differs from the one in $\mathcal{G}_{n}^{\prime}$ by $\gamma$. Finally, for any $k>n$, any cell of $\mathcal{G}_{k}^{\prime}$, and any lift of its boundary, we add a single extended lift to $\tilde{\mathcal{G}}_{k}$ if one exists.

Now we modify $\tilde{H} \circ G^{\prime}$ to define a map $\left.\tilde{F}\right|_{(X \times[0,1])^{(n)}}$ : for every $n$-cell $c$, we let the map on $c$ be $\ell\left(\left.\tilde{H} \circ G^{\prime}\right|_{c},-\mathfrak{b}(c)\right)$. This kills the obstruction, allowing us to lift further to construct our $\tilde{\mathcal{G}}$-mosaic $\operatorname{map} \tilde{F}: X \times[0,1] \rightarrow Y$.

\section{RATiOnAL INVARIANCE}

In this section, we show that the difficulty of nullhomotoping $L$-Lipschitz maps $X \rightarrow Y$ depends on $Y$ only up to rational homotopy type. The proof of this can be separated into a topological result and a metric result.

We start with the metric result, which is again proven in CDMW. It shows that if a map $X \rightarrow Z$ is homotopically trivial relative to a subspace $Y \subset Z$ whose relative homotopy groups are finite, then one can find such a homotopic trivialization which is geometrically bounded.

Lemma 5.1. Let $Y \subset Z$ be a pair of finite simplicial complexes such that $\pi_{k}(Z, Y)$ is finite for $k \leq n+1$. Then there is a constant $C(n, Y, Z)$ with the following property. Let $(X, A)$ be a pair of (not necessarily finite) $n$-dimensional simplicial complexes and $f:(X, A) \rightarrow(Z, Y)$ a simplicial map which is homotopic rel $A$ to a map $g: X \rightarrow Y$. Then there is a short homotopy rel $A$ of $f$ to a map $g^{\prime}$ which lands in $Y$ and is homotopic as a map into $Y$ to $g$. By "short", we mean that it is $C$-Lipschitz under the standard metric on the product cell structure on $X \times[0,1]$.

Note that the constant $C$ does not depend on $X$ and in particular on the choice of a subdivision of $X$. Thus if we consider Lipschitz and not just simplicial maps from $X$ to $Y$, the width of the homotopy remains constant, rather than linear in the Lipschitz constant.

We now move on to the topological portion of the discussion, in which we prove Theorem 1.4 . First we state this result more precisely. 


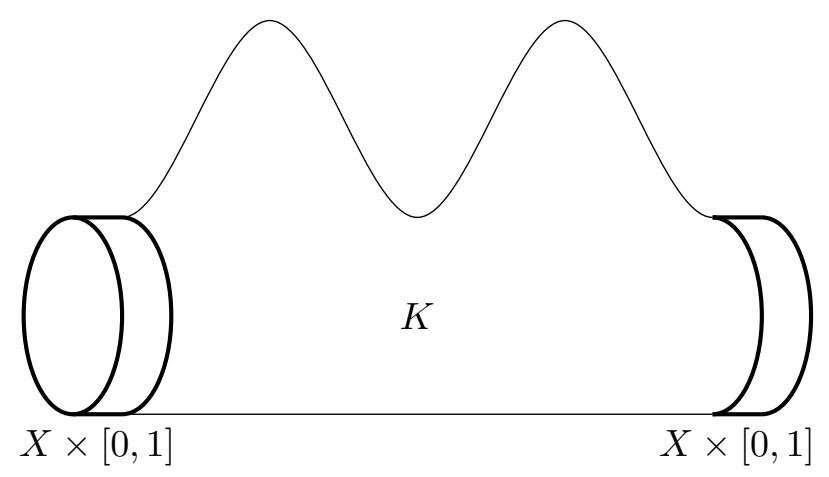

Figure 1. A camel. Note the two collars which are isometric to some fixed simplicial structure on $X \times[0,1]$.

Theorem 5.2. Rationally equivalent simply connected finite simplicial complexes admit nullhomotopies of the same shapes. That is, suppose we are given the following data:

(1) Rationally homotopy equivalent simply connected finite metric simplicial complexes $Y$ and $Z$;

(2) A finite $n$-dimensional simplicial complex $X$;

(3) A simplicial pair $(K, X \times([0,1] \cup[2,3]))$ which is homeomorphic to

$$
(X \times[0,3], X \times([0,1] \cup[2,3]))
$$

and given the standard metric on simplices. Here the product of $X$ with each unit interval is given an arbitrary fixed simplicial structure which restricts at $t=0$ and $t=1$ to the simplicial structure on $X$.

Then there is a constant $C=C(X, Y, Z)>0$ such that if for every nullhomotopic L-Lipschitz map $f: X \rightarrow Y$ there is an $M$-Lipschitz nullhomotopy $F: K \rightarrow Y$, then for every L/C-Lipschitz map $g: X \rightarrow Z$ there is a $(C M+C)$-Lipschitz nullhomotopy $G: K \rightarrow Z$.

The point of introducing the complex $K$ is to prescribe a metric on the cylinder $X \times[0,3]$. The theorem then says that, under any such metric, sizes of homotopies do not depend very much on torsion in the target space. For example, this controls the sizes of nullhomotopies through a camel with two humps, as in the figure. In the main application of this theorem to the proof of Theorem 1.2. $K$ will be a straight, but elongated cylinder whose length depends on the the Lipschitz constant.

Proof. Since $Y$ and $Z$ are rationally homotopy equivalent, there is a finite complex $W$ and a pair of maps $Y \rightarrow W \leftarrow Z$ which induce equivalences on the level of rational homotopy. A proof for this is given, for example, in [Ma, Lemma 1.3 and Cor. 1.9]. Thus we can assume that $Y$ is a subcomplex of $Z$ or vice versa.

We first do the case when $Y \subset Z$. Let $C(n, Y, Z)$ be the constant given in Lemma 5.1. Suppose $g: X \rightarrow Z$ is a nullhomotopic $L / C$-Lipschitz map, which we can assume to be simplicial on a subdivision $X_{L}$ at scale $\sim C / L$. In particular, $g$ can be homotoped into $Y$, and so by Lemma 5.1 this can be done via a short homotopy $H: X_{L} \times[0,1] \rightarrow Z$. Now, $f(x):=H(x, 1)$ is an $L$-Lipschitz nullhomotopic map $X \rightarrow Y$, and so there is an $M$-Lipschitz nullhomotopy $F: K \rightarrow Y$ of $f$. Concatenating $H$ and $F$ gives an $(M+C)$-Lipschitz nullhomotopy $G: K \rightarrow Z$ of $g$. This completes the first case.

Now suppose $Z \subset Y$, and suppose $g: X \rightarrow Z$ is a nullhomotopic $L$-Lipschitz map. By assumption, there is an $M$-Lipschitz nullhomotopy $F: K \rightarrow Y$ of $g$ (as a map to $Y$ ) and an uncontrolled nullhomotopy $\tilde{G}: C X \rightarrow Z$ of $g$. There is no guarantee, however, that $F$ can be homotoped into $Z \operatorname{rel} X$, even in an uncontrolled way. 
On the other hand, concatenating $F$ and $\tilde{G}$ along $g$ gives us a map $H: S X \rightarrow Y$. Homotopy classes of such maps form a group, and the induced mapping $[S X, Z] \rightarrow[S X, Y]$ is a homomorphism. We would like to analyze the cokernel of this homomorphism; to do this, we use obstruction theory on the relative Postnikov tower

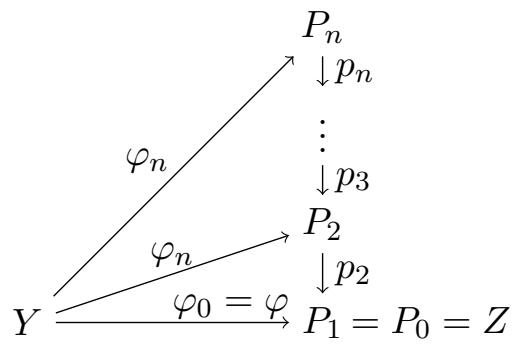

of the inclusion $\varphi: Y \hookrightarrow Z$. Here, $P_{k}$ is a space such that $\pi_{i}\left(P_{k}, Y\right)=0$ for $i \leq k$ and $\pi_{i}\left(Z, P_{k}\right)=0$ for $i>k$. The map $p_{k}$ therefore only has one nonzero relative homotopy group, $\pi_{k}(Z, Y)$. In this setting there is an obstruction theory long exact sequence of groups

$$
\cdots \rightarrow H^{k-1}\left(X ; \pi_{k}(Z, Y)\right) \rightarrow\left[S X, P_{k}\right] \rightarrow\left[S X, P_{k-1}\right] \rightarrow H^{k}\left(X ; \pi_{k}(Z, Y)\right) \rightarrow \cdots .
$$

Thus the cokernel we are interested in has cardinality at most $\prod_{i=1}^{n}\left|H^{k}\left(X ; \pi_{k}(Z, Y)\right)\right|$. For each element $\gamma$ of this cokernel, choose a map $F_{\gamma}: S X \rightarrow Y$ representing it.

Now let $R \subset K=X \times(5 / 2,3]$. Then there is an obvious 2-Lipschitz homeomorphism $\psi_{1}$ : $K \backslash R \rightarrow K$ which is the identity outside $X \times[2,3]$. Also, let $\psi_{2}: \bar{R} \rightarrow S X$ be the surjection which contracts $X \times\{5 / 2\}$ and $X \times\{3\}$. Then the map

$$
\tilde{F}(x)= \begin{cases}F \circ \psi_{1}(x) & x \in K \backslash R \\ F_{-[H]} \circ \psi_{2}(x) & x \in R\end{cases}
$$

gives us a nullhomotopy of $g$ which can be homotoped into $Z$ and which is $\left(C_{0} M+C_{0}\right)$-Lipschitz, where $C_{0}$ depends only on the geometry of the various $F_{\gamma}$.

Finally, we can use Lemma 5.1 to ensure that we get a $(C M+C)$-Lipschitz nullhomotopy $G: K \rightarrow Z$, where $C$ is the product of $C_{0}$ and the constant from the lemma.

\section{Proof of the MAIN THEOREM}

Putting together Theorems 5.2 and 4.1, we can now prove Theorem 1.2. We restate this theorem equivalently below:

Theorem. Let $X$ be an n-dimensional finite complex, and let $Y$ be a finite complex which is rationally equivalent up to dimension $n$ to the total space of an induced fibration whose fiber and base are both products of simply connected Eilenberg-MacLane spaces. Then there is a constant $C(X, Y)$ such that nullhomotopic L-Lipschitz maps from $X$ to $Y$ admit nullhomotopies of thickness $C(L+1)$ and width $C(L+1)^{2}$.

Let's unwrap a bit the rational homotopy theory of the spaces that we are considering, particularly the word induced.

The basic fact that underlies everything is that $H^{*}(K(\mathbb{Q}, k) ; \mathbb{Q})=\mathbb{Q}[x]$ if $k$ is even and is $\mathbb{Q}[x] /\left(x^{2}=0\right)$ if $k$ is odd. Note that both cases can be described as saying that the cohomology is the free graded-commutative differential algebra on a $k$-dimensional class. In light of Kunneths theorem, we can now say that if $V$ is a graded vector space, and $K(V)$ is a product of EilenbergMacLane spaces $K\left(V_{k}, k\right)$, then the rational cohomology of $K(V)$ is the free graded algebra $\mathbb{Q}[V]$.

Notice of course, that if $X$ is a space whose rational cohomology is a free DGA, then by considering the generating cohomology classes as maps into Eilenberg-MacLane spaces, we get a map into a product of such spaces, i.e. a map $X \rightarrow K(V)$ which is a rational isomorphism. 
A special case is the even dimensional sphere. $S^{2 k} \rightarrow K(\mathbb{Z}, 2 k)$ is a tautological map. However, the cup square vanishes for the sphere for dimensional reasons, so this tautological map lifts naturally to the homotopy fiber of the map $K(\mathbb{Z}, 2 k) \stackrel{\cup 2}{\longrightarrow} K(\mathbb{Z}, 4 k)$. The map to this fiber is a rational equivalence, as seen using the fact that the Euler class of the rational fibration sequence

$$
S_{(0)}^{4 k-1}=K(\mathbb{Q}, 4 k-1) \rightarrow S_{(0)}^{2 k} \rightarrow K(\mathbb{Q}, 2 k)
$$

is cup square together with the Gysin sequence.

More general homogeneous spaces $G / H$ have similar structure (after some work!) The inclusion $H \rightarrow G$ is a homomorphism, and therefore induces a map $B H \rightarrow B G$ whose fiber is easily seen to be $G / H$. For any connected Lie group $K$, the cohomology is a free algebra, i.e., since $K$ is finitedimensional, it is the cohomology of a product of odd spheres; by a theorem of Hopf, this product is in fact homotopy equivalent to $K$ (see e.g. Example 3 of [FHT, $\S 12(\mathrm{a})]$ ). That the cohomology of $B K$ is also free is less obvious, but is also classical; this cohomology can be described using the Lie algebra of $K$. This gives rise to a description of the map $B H \rightarrow B G$, which also shows that the map $G / H \rightarrow B H$ is (up to homotopy) an induced fibration, a notion we now explain in our setting. A proof of this can be found in [FHT, $\S 15(\mathrm{f})]$.

Suppose now that we have two graded $\mathbb{Q}$-vector spaces $V$ and $W$. To describe a map $f: K(V) \rightarrow$ $K(W)$ is the same thing as describing a graded homomorphism $W \rightarrow \mathbb{Q}[V]$. The fiber $F$ of this map has a description via a fibration

$$
K(W,[-1]) \rightarrow F \rightarrow K(V) .
$$

(where the $[-1]$ indicates a shift in grading by -1 ), but it is not the most general such fibration; we say, following Ganea, that it is induced (by the map $K(V) \rightarrow K(W)$. In the general case, the structure group would be a space of self-homotopy equivalences of $K(W,[-1])$, but here we are only allowing $K(W,[-1])$ itself, acting on itself as a topological group. The classifying space of $K(W,[-1])$ is, of course $K(W)$.

In this case, the free algebra generated by $W$ with the shifted grading together with $V$, equipped with the differential given by $d w=f^{*} w$, is a DGA model for the space $F$. A minimal model for this DGA is obtained by deleting pairs of indecomposables (that is, elements of $W$ and $V)(g, h)$ with $d g=h$. Conversely, given such a minimal model, we can construct an induced fibration using the recipe above. This shows that this condition is equivalent to that in the introduction.

Moreover, by choosing a lattice $V_{\mathbb{Z}} \subset V$ and a lattice in $W$ whose differential lands in $\mathbb{Q}\left[V_{\mathbb{Z}}\right]$, one constructs an induced fibration of this form whose homotopy groups are all free abelian and whose total space has finite skeleta. We use this construction in the proof below.

Thus the space $Y$ in Theorem 1.2 can be any simply-connected homogeneous target space, including spheres, complex projective spaces, and Grassmannians. Another corollary concerns maps to spaces which are highly connected. The first part is a result from [CDMW].

Corollary 6.1. Let $Y$ be a rationally $(k-1)$-connected finite complex and $X$ an $n$-dimensional finite complex.

(a) If $n \leq 2 k-2$, then there is a constant $C(X, Y)$ such that homotopic L-Lipschitz maps from $X$ to $Y$ admit $C(L+1)$-Lipschitz homotopies.

(b) If $2 k-1 \leq n \leq 3 k-3$, then there is a constant $C(X, Y)$ such that nullhomotopic L-Lipschitz maps admit nullhomotopies of thickness $C(L+1)$ and width $C(L+1)^{2}$.

Proof of Theorem 1.2. As discussed above, $Y$ is rationally equivalent up to dimension $n$ to the total space $Z$ of an induced fibration

$$
\prod_{j=1}^{s} K\left(\mathbb{Z}, n_{j}\right) \rightarrow Z \rightarrow \prod_{i=1}^{r} K\left(\mathbb{Z}, n_{i}\right) .
$$


As noted before, we may assume that $Z$ is the fiber product of fibrations $K\left(\mathbb{Z}, n_{j}\right) \rightarrow Z_{j} \rightarrow B$, where $B$ is as in Theorem 4.1 and the $Z_{j}$ have finite skeleta. Concretely, we can think of $Z$ as the pullback via the diagonal map $B \rightarrow B^{s}$ of the product fibration

$$
Z_{1} \times \cdots \times Z_{s} \rightarrow B^{s} .
$$

Then if $f$ is a nullhomotopic map $X \rightarrow Z$, we can construct a nullhomotopy with the desired properties by finding a nullhomotopy $F$ in $B$, lifting it as in Theorem 4.1 to $\tilde{F}_{j}: X \times I \rightarrow Z_{j}$ for each $1 \leq j \leq s$, and finally setting

$$
\tilde{F}(x, t)=\left(\tilde{F}_{1}(x, t), \ldots, \tilde{F}_{s}(x, t)\right) \in Z .
$$

Once we have shown the result for $Z$, it must hold for $Y$ by Theorem [5.2, as follows. For a given $L$, we take $K_{L}=X \times[0, L]$. We have shown that for any nullhomotopic $L$-Lipschitz $f: X \rightarrow Z$, there is a $C L$-Lipschitz nullhomotopy of $f$ in $K_{L}$. Therefore, the same is true in $Y$, with a different constant. Compressing $K_{L}$ back down to $X \times[0,1]$, we get back our separate estimates on thickness and width.

\section{SOME LOWER BOUNDS}

It is first worth noting that nullhomotopies of maps, for example, from $S^{n} \rightarrow S^{n}$ and $S^{3} \rightarrow \mathbb{C P}^{2}$ cannot be done in constant time, as is the case for targets with finite homotopy groups as in Theorem 1 of [FW]. Thus the linear upper bound in Conjecture 3 when $q=1$ is sharp. All this is discussed in [CDMW].

One may ask then whether Theorem 1.2 similarly gives sharp bounds. This boils down to two separate questions. First, is the quadratic bound on the width of the homotopy necessary, or could a linear bound suffice? Secondly, can the theorem be extended to homotopies rather than just nullhomotopies, as is the case with the theorem in [CDMW]? It turns out that both of these features are required.

7.1. Maps that are hard to nullhomotope. First, we give a series of examples (see also CDMW] that show that for every $q$, the upper bound in Conjecture 3 is the best one possible in general. In particular, we show that it gives a sharp estimate on the minimum volume of a nullhomotopy in certain cases; this can potentially be apportioned to the width and thickness in other ways. Let the space $Y_{q}$ be given by $S^{2} \vee S^{2}$ together with $(q+3)$-cells whose attaching maps form a basis for $\pi_{q+2}\left(S^{2} \vee S^{2}\right) \otimes \mathbb{Q}$. Note that by rational homotopy theory, $\pi_{*+1}\left(S^{2} \vee S^{2}\right) \otimes \mathbb{Q}$ is a free graded Lie algebra on two generators of degree 1 whose Lie bracket is the Whitehead product. In particular, if $f$ and $g$ are the identity maps on the two copies of $S^{2}$, the iterated Whitehead product

$$
h_{1}=[f,[f, \ldots[f, g] \ldots]]: S^{q+2} \rightarrow S^{2} \vee S^{2},
$$

with $f$ repeated $q$ times, represents a nonzero element of $\pi_{q+2}\left(S^{2} \vee S^{2}\right)$. Moreover, the map

$$
h_{L}=\left[L^{2} f,\left[L^{2} f, \ldots\left[L^{2} f, L^{2} g\right] \ldots\right]\right]: S^{q+2} \rightarrow S^{2} \vee S^{2}
$$

is an $O(L)$-Lipschitz representative of $L^{2 q+2}\left[h_{1}\right]$. Thus in $Y_{q}$, we can define a nullhomotopy $H$ of $h_{L}$ by first homotoping it inside $S^{2} \vee S^{2}$ to $h_{1} \circ \varphi_{2 q+2}$ for some map $\varphi_{2 q+2}: S^{q+2} \rightarrow S^{q+2}$ of degree $L^{2 q+2}$, and then nullhomotoping each copy of $h_{1}$ via a standard nullhomotopy.

Since $h_{1}$ is not nullhomotopic in $S^{2} \vee S^{2}$, this standard nullhomotopy must have degree $C \neq 0$ (in the sense of relative homology) on at least one of the $(q+3)$-cells, giving a closed $(q+3)$-form $\omega$ on $Y$ such that $\int_{S^{q+2} \times I} \omega^{*} H=L^{2 q+2} C$. Now, suppose $H^{\prime}$ is some other nullhomotopy of $h_{L}$. Then gluing $H$ and $H^{\prime}$ along the copies of $S^{q+2} \times\{0\}$ gives a map $p: S^{q+3} \rightarrow Y$. Since the Hurewicz map sends $\pi_{q+3}\left(Y_{q}\right)$ to zero, the total degree of $p$ on cells must be zero. This shows that $p$ must 


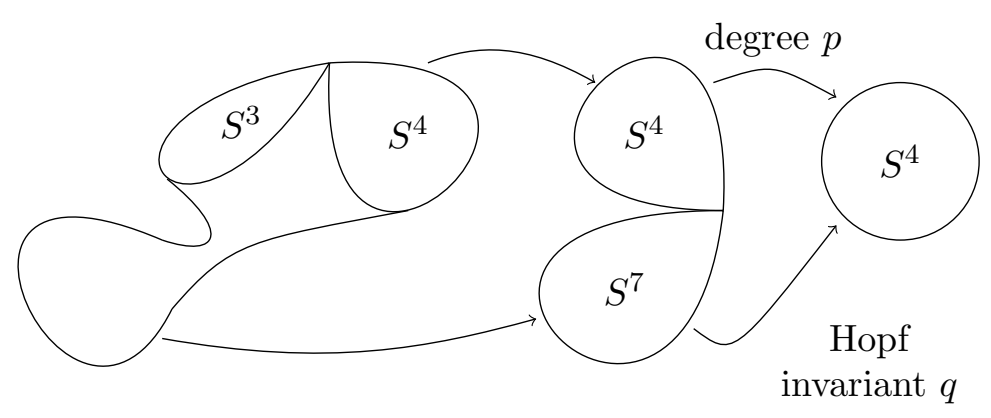

Figure 2. Construct maps $S^{3} \times S^{4} \rightarrow S^{4}$ by "budding off" a small ball and then projecting the rest onto the $S^{4}$ factor.

have degree zero on cells, in other words, that $\int_{S^{q+2} \times I} \omega^{*} H^{\prime}=L^{2 q+2} C$. Thus the volume of a nullhomotopy of $h_{L}$ grows at least as $L^{2 q+2}$.

In particular, a nullhomotopy $F: S^{q+2} \times[0,1] \rightarrow Y_{q}$ of $h_{L}$ which has thickness $\sim L$ has to have width $\sim L^{q}$.

Now, the rational homotopy groups of $Y_{q}$ are given by the free Lie algebra on two generators truncated in degree $q+1$. A standard computation shows that differentials of $r$-dimensional generators in the corresponding minimal model are multiples of the $(r-1)$-dimensional generators. Therefore, the minimal depth of the filtration in Conjecture 3 in this case is $q$, demonstrating that this is in some sense the "best possible" conjecture. In particular, the bound in Theorem 1.2 is sharp in at least some cases. On the other hand, it is still open whether this quadratic bound is sharp, for example, for maps $S^{3} \rightarrow S^{2}$.

7.2. Maps that are hard to homotope. To see that general homotopies do not always behave like nullhomotopies, we consider maps $S^{3} \times S^{4} \rightarrow S^{4}$. Any map $f: S^{3} \times S^{4} \rightarrow S^{4}$ induces a homomorphism of minimal DGAs

$$
\left\langle x^{4}, y^{7}: d x=0, d y=x^{2}\right\rangle \stackrel{f^{*}}{\rightarrow}\left\langle a^{3}, b^{4}, c^{7}: d a=d b=0, d c=b^{2}\right\rangle
$$

which must send $x \mapsto p b$ and $y \mapsto p^{2} c+q a b$ for some $p, q \in \mathbb{Q}$. Conversely, for any $p, q \in \mathbb{Z}$ we can define a map $f_{p, q}$ as illustrated in Figure 2 such that $f_{p, q}^{*}$ sends $x \mapsto p b$ and $y \mapsto p^{2} c+q a b$. This follows from the action of the first map on cohomology and of the second on homotopy groups.

Now, given $p \neq 0$, for any $q_{1}, q_{2} \in \mathbb{Q}$ there is a homotopy of DGA homomorphisms

$$
\left\langle x^{4}, y^{7}: d x=0, d y=x^{2}\right\rangle \rightarrow\left\langle a^{3}, b^{4}, c^{7}: d a=d b=0, d c=b^{2}\right\rangle \otimes\left\langle t^{0}, d t^{1}\right\rangle
$$

between $f_{p, q_{1}}^{*}$ and $f_{p, q_{2}}^{*}$, given by

$$
\begin{aligned}
& x \mapsto p b+\frac{q_{2}-q_{1}}{2 p} a d t \\
& y \mapsto p^{2} c+q_{1} a b(1-t)+q_{2} a b t .
\end{aligned}
$$

This suggests that, at least up to a finite order difference, $f_{p, q_{1}} \simeq f_{p, q_{2}}$.

Indeed, one can see more geometrically that two such maps are homotopic if the number $\frac{q_{2}-q_{1}}{2 p}$ is an integer. For concreteness, suppose $p=1$ and $q_{1}=0$. A potential homotopy between $f_{1,0}$ and $f_{1, q}$ must factor through the quotient space of $S^{3} \times S^{4} \times I$ where $S^{3} \times S^{4} \times\{0\}$ is projected onto $S^{4}$ and $S^{3} \times S^{4} \times\{1\}$ is mapped onto $S^{4} \vee S^{7}$ as in Figure 2, This quotient space is easily seen to be homeomorphic to $S^{4} \times S^{4}$ minus an open ball, and thus homotopy equivalent to $S^{4} \vee S^{4}$. Let $\alpha$ and $\beta$ be the homotopy classes of the identity maps on the copies of $S^{4}$, which are images under the quotient map of $S^{3} \times S^{4} \times\{0\}$ and $S^{3} \times * \times I$ respectively. Since we know what happens on 
the ends of the interval, we see that, if $h: S^{4} \vee S^{4} \rightarrow S^{4}$ is a map in the homotopy class of such a homotopy, then $h_{*} \alpha=\left[\operatorname{id}_{S^{4}}\right]$ and

$$
h_{*}[\alpha, \beta]=\frac{q}{2}\left[\operatorname{id}_{S^{4}}, \operatorname{id}_{S^{4}}\right]=q[\text { Hopf }] .
$$

Indeed such a map $h$ exists, with $h_{*} \beta=\frac{q}{2}\left[\operatorname{id}_{S^{4}}\right]$. In other words, there is a homotopy $F: S^{3} \times S^{4} \times$ $I \rightarrow S^{4}$ between $f_{1,0}$ and $f_{1, q}$, and any such homotopy satisfies $\int_{S^{3} \times * \times I} F^{*} d$ vol $=\frac{q}{2}$.

If we take $q=L^{8}$, the way we have defined $f_{1, q}$ gives it Lipschitz constant $O(L)$. On the other hand, we have just shown that a homotopy between $f_{1, L^{8}}$ and $f_{1,0}$ must have degree at least $L^{8} / 2$ on the 4-dimensional submanifold $S^{3} \times * \times I \subset S^{3} \times S^{4} \times I$. Thus such a homotopy must have Lipschitz constant $\Omega\left(L^{2}\right)$, or, if it has linear thickness, it must have width $\Omega\left(L^{5}\right)$. Either way, it cannot possibly satisfy the bounds of Theorem 1.2, showing that the theorem cannot directly generalize beyond nullhomotopies.

\section{REFERENCES}

[Amann] Manuel Amann, Degrees of self-maps of simply connected manifolds, International Mathematics Research Notices (2014), doi:10.1093/imrn/rnu201.

[BMSS] Richard Body, Mamoru Mimura, Hiroo Shiga, and Dennis Sullivan, p-universal spaces and rational homotopy types, Commentarii Mathematici Helvetici 73 (1998), no. 3, 427-442.

[CDMW] Gregory R. Chambers, Dominic Dotterrer, Fedor Manin, and Shmuel Weinberger, Quantitative nullcobordism, arXiv preprint arXiv:1610.04888 (2016).

[EdGr] Herbert Edelsbrunner and Daniel R. Grayson, Edgewise subdivision of a simplex, Discrete \& Computational Geometry 24 (2000), no. 4, 707-719.

[FHT] Yves Félix, Steve Halperin, and Jean-Claude Thomas, Rational homotopy theory, Graduate Texts in Mathematics, vol. 205, Springer, 2012.

[FW] Steve Ferry and Shmuel Weinberger, Quantitative algebraic topology and lipschitz homotopy, Proceedings of the National Academy of Sciences 110 (2013), no. 48, 19246-19250.

[Ganea] Tudor Ganea, Induced fibrations and cofibrations, Transactions of the American Mathematical Society 127 (1967), no. 3, 442-459.

[Gro78] Mikhail Gromov, Homotopical effects of dilatation, Journal of Differential Geometry 13 (1978), no. 3, $303-310$.

[Gro99] Q Quantitative homotopy theory, Invited Talks on the Occasion of the 250th Anniversary of Princeton University (H. Rossi, ed.), Prospects in Mathematics, 1999, pp. 45-49.

[Gro14] _ Manifolds: Where do we come from? what are we? where are we going?, The Poincaré conjecture (James Carlson, ed.), Clay Mathematics Proceedings, vol. 19, 2014, pp. 81-144.

[Ma] Fedor Manin, Volume distortion in homotopy groups, Geometric and Functional Analysis 26 (2016), no. 2, $607-679$.

[Mil] John Milnor, Construction of universal bundles, I, Annals of Mathematics 63 (1956), no. 2, 272-284.

[MiT] Mamoru Mimura and Hirosi Toda, On p-equivalences and p-universal spaces, Commentarii Mathematici Helvetici 46 (1971), no. 1, 87-97.

[Whi] Hassler Whitney, Geometric integration theory, Princeton Mathematical Series, vol. 21, Princeton University Press, 1957.

Department of Mathematics, University of Chicago, Chicago, Illinois, USA

E-mail address, G. R. Chambers: chambers@math.uchicago.edu

Department of Mathematics, University of Toronto, Toronto, Ontario, Canada

E-mail address, F. Manin: manin@math.toronto.edu

Department of Mathematics, University of Chicago, Chicago, Illinois, USA

E-mail address, S. Weinberger: shmuel@math.uchicago.edu 